\title{
Caracterización de la ubicación de madrigueras de Simosciurus nebouxii en un área urbana de Lima, Perú
}

\author{
Nest site characterization of Simosciurus nebouxii in an urban area of Lima, Peru
}

\author{
Leticia Lajo-Salazar $^{1 \star \star}$, Marta Williams León de Castro ${ }^{2}$ \\ y Pedro Vásquez Ruesta ${ }^{3}$
}

\begin{abstract}
Resumen
La ardilla de nuca blanca Simosciurus nebouxii (antes Sciurus stramineus) es nativa de la costa norte de Perú y ha sido introducida en la ciudad de Lima en las últimas décadas. $\mathrm{Su}$ éxito en este ámbito es evidencia de la alta plasticidad característica de las ardillas arborícolas. Sin embargo la información sobre su ecología es muy limitada. Para aportar al conocimiento de los aspectos ecológicos básicos de la especie, se analizó la ubicación de 33 madrigueras en el campus de la Pontificia Universidad Católica del Perú, describiendo las áreas que las rodeaban, los árboles que las sostenían y la posición en la copa del árbol. Las áreas tuvieron una densidad promedio de 37.5 árboles/ha y una cubierta de dosel de entre 33 y 75\%. Las madrigueras estuvieron ubicadas en árboles de 6 géneros distintos, pero fueron 3 de ellos los que agruparon más del 80\% de las madrigueras (Tipuana, Fraxinus y Eucalyptus). Dichos árboles tuvieron en promedio $11.8 \mathrm{~m}$ de altura, $94.2 \mathrm{~m}^{2}$ de área de copa y $67.7 \%$ de cobertura de copa. Además, las madrigueras estuvieron situadas a $9.1 \mathrm{~m}$ de altura en promedio y se identificó un patrón en la posición relativa de las madrigueras siguiendo la forma de las copas de los árboles donde se encuentran y ligeramente por debajo del borde.
\end{abstract}

Palabras clave: ardilla arborícola, cobertura reproductiva, fauna urbana, madrigueras, Perú.

\footnotetext{
${ }^{1}$ División de Mastozoología, Centro de Ornitología y Biodiversidad (CORBIDI), Santa Rita Nº105 Of. 2, Urb. Huertos de San Antonio, Surco, Lima, Perú.

${ }^{2}$ Laboratorio de Fisiología Animal y Biorremediación Luis Basto Acosta, Facultad de Ciencias, Universidad Nacional Agraria La Molina (UNALM), Av. La Molina s/n, La Molina, Lima, Perú.

${ }^{3}$ Centro de Datos para la Conservación, Facultad de Ciencias Forestales, Universidad Nacional Agraria La Molina (UNALM), Av. La Molina s/n, La Molina, Lima, Perú.

* Autor de Correspondencia: leticia.lajo@gmail.com
} 


\begin{abstract}
The white-napped squirrel Simosciurus nebouxii (formerly known as Sciurus stramineus) a native species of northern Peru, has been recently introduced to Lima city. The success of its adaptation to the new habitat is clear evidence of the high plasticity of tree squirrels. Even though that now it is a common species there is scarce information about its ecological patterns. To contribute to the knowledge on some basic ecology of this species we analyzed the location of 33 squirrel nests at the Pontificia Universidad Católica del Perú. Describing the nest surrounding areas, the trees where the nests were placed, and the position of the nest on the trees. The areas surrounding the nests had 37.5 trees/hectare in average, and a canopy closure from 33 to $75 \%$. Nests were placed on trees of six different genera, but three of them (Tipuana, Fraxinus and Eucalyptus) represented more than $80 \%$ of the nests. The average values of the variables evaluated on trees with nests were: $11.8 \mathrm{~m}$ height, $94.2 \mathrm{~m}^{2}$ of treetop area, and $67.7 \%$ of treetop cover. Furthermore, the nests were placed at $9.1 \mathrm{~m}$ high on average, and we observed a pattern of the relative position of nests following the treetop shape just below the edge.
\end{abstract}

Key words: tree squirrel, breeding cover, urban wildlife, squirrel nest sites, Peru.

\section{Introducción}

La revisión de la taxonomía de la familia Sciuridae en Sudamérica realizada por De Vivo y Carmignotto (2015) propone una reasignación de géneros para las ardillas de la región Neotropical, así como una división de la especie conocida como Sciurus stramineus (Eydoux \& Souleyet, 1841). El rango de distribución de dicha especie se extendía desde el extremo noroeste de Perú, hasta la parte central del este de Ecuador (Emmons y Feer 1997; Tirira 2007; Pacheco et al. 2009; Thorington et al. 2012). Sin embargo, ahora se reconocen dos especies de morfología similar que, hasta donde se sabe, se encuentran distribuidas de forma alopátrica en dicha área (De Vivo y Carmignotto 2015). Estas son Simosciurus stramineus (P. Gervais, 1841) y Simosciurus nebouxii (I. Geoffroy St.Hilaire, 1855). La última, también conocida como ardilla de nuca blanca, se distribuye desde el extremo sudeste de Ecuador, provincias de El Oro y Loja, hasta Perú, departamentos de Tumbes, Piura y Lambayeque. Es la única especie de esciúridos en el Perú que habita en bosques secos, siendo los demás miembros de la familia propios de los bosques húmedos tropicales (Pacheco et al. 2009; De Vivo y Carmignotto 2015). Aunque las ardillas arborícolas son comunes en muchas localidades del
Perú, la investigación sobre ellas es muy escasa (Koprowski y Nandini 2008; Jessen 2013; Jessen et al. 2013).

La ardilla de nuca blanca fue introducida en la ciudad de Lima en la década del 70, primero en el área del Zoológico Parque de Las Leyendas (Jessen et al. 2010) y posiblemente en sucesivos eventos correspondientes a escapes o liberaciones de individuos inicialmente mantenidos como mascotas. Su población ha aumentado hasta hacerse común en parques y calles arboladas (Jessen et al. 2010; Merrick et al. 2012), tal como ha ocurrido con especies similares en otras regiones del mundo (Long 2003). Sin embargo, la información sobre su ecología es casi inexistente, lo que podría limitar el manejo de su población de ser necesario. Más aun cuando los escasos estudios realizados para esta especie en la ciudad de Lima, por Montes et al. (2011) y Díaz y Tantaleán (2012) enfocados en aspectos de salud, concluyeron que podrían constituir un riesgo por ser éstas reservorios de leptospirosis y hospederos de parásitos intestinales, respectivamente. Por tal motivo, el objetivo del presente estudio es aportar al conocimiento de los aspectos básicos de la forma de vida de esta ardilla, como es la ubicación de las madrigueras, lo que da luces sobre las características de la cobertura reproductiva en un área urbana. 


\section{Materiales y Métodos}

La Pontifica Universidad Católica del Perú (PUCP) se ubica en el distrito de San Miguel, provincia de Lima, departamento de Lima. El campus cuenta con 41 hectáreas de las cuales 17 correspondían a áreas verdes a la fecha de la evaluación, las cuales albergaban al menos 40 especies de árboles (Sotomayor com. pers. ${ }^{1}$ ), número que se ha ido incrementando notablemente los últimos años gracias a la implementación de espacios que buscan representar diferentes hábitats del Perú y del mundo como el "bosque seco tropical", el "bosque húmedo tropical”, el "jardín de palmeras" y el "jardín de baobabs”, entre otras áreas verdes (PUCP 2018)

En él habitan un grupo de venados de cola blanca Odocoileus virginianus que se encuentran custodiados por la institución bajo la figura de zoocriadero, con licencia del Servicio Nacional Forestal y de Fauna Silvestre desde el año 2010 (Ruiz et al. 2014). También es hábitat de numerosas especies de aves, entre las que se han podido observar el turtupilín (Pyrrocephalus rubinus), la amazilia costeña (Amazilia amazilia), la cuculí (Zenaida meloda), el loro cabeza roja (Aratinga wagleri), el cucarachero (Troglodytes aedon), el mielerito (Coereba flaveola), el botón de oro (Sicalis flaveola), el tordo parasito (Molothrus bonaerensis), el violinista (Thraupis episcopus) y el gavilán acanelado (Parabuteo unicinctus). Siendo la ardilla competidora de algunas de las aves mencionadas y presa de la última.

Durante los meses de abril y mayo de 2013 se realizaron recorridos cubriendo todas las áreas verdes del campus en busca de madrigueras. Se registró la posición de cada árbol con madriguera, considerando solamente las madrigueras activas, reconociéndose como tales aquellas en las que no se pudiera ver la luz a través de su estructura (Raspopov e Isakov 1980; Salsbury 2008).

Entre los días 1ro y 21 de mayo se registraron los valores de cada variable a evaluarse, sepa-

\footnotetext{
${ }^{1}$ Sotomayor, C. 23 mar. 2012. Presencia de ardillas en el campus de la PUCP (entrevista). Lima, Perú, Pontificia Universidad Católica del Perú.
}

radas en tres escalas: (A) área circundante de la madriguera, (B) árbol con madriguera y (C) posición de la madriguera en el árbol. Dichas escalas se determinaron bajo el criterio jerárquico de la selección de hábitat (Rotenberry y Wiens 1980; Knick y Rotenberry 1995) y siguiendo estudios relacionados en otras especies de esciúridos (Edelman y Koprowski 2005; Merrick et al. 2007; Ramos-Lara y Cervantes 2007; Cudworth y Koprowski 2011).

La primera escala (A) se definió como una parcela circular de 50 metros de diámetro cuyo centro era el árbol que sostenía la madriguera en evaluación. En ella se midió la densidad de árboles con una altura mínima de 5 metros y diámetro a la altura del percho (DAP) igual o mayor a $20 \mathrm{~cm}$ (DEN), el número de árboles adicionales con madriguera (NAA), el número de árboles o arbustos con alguna parte que constituya alimento para la especie (NAC), el número de árboles o arbustos con materiales para la construcción de madrigueras (NAM), la cobertura de dosel (COD) y la proporción de área edificada (PAE). En la segunda escala (B) se describieron atributos del árbol, considerando la especie, el número de madrigueras que posee (NMA), el DAP, la altura total (ATA), la altura mínima de copa (AMC), la amplitud de copa (ACA) y la cobertura de copa (CCA). En la tercera escala $(C)$ se describió la posición de la madriguera en la estructura tridimensional del árbol, considerándose la altura (ALM), la distancia al eje del árbol (DEA), la distancia al borde de la copa (DBC) y la orientación cardinal respecto al eje del árbol. Para las medidas de longitud y distancia se utilizó una cinta métrica de $30 \mathrm{~m}$, para las medidas de altura se utilizó un clinómetro y para las medidas de cobertura se utilizó un densiómetro, evaluando un total de 20 puntos ( 5 por cada dirección cardinal) separados de forma equidistante en las áreas y por debajo de las copas dependiendo del tamaño del área que se cubriera, y se anotó con 1, para 'cobertura', y 0 para 'sin cobertura', hallándose el porcentaje total de cobertura (\%) sumando los valores encontrados y dividiéndolo entre veinte. Las especies vegetales que se considera que constituyen alimento (Cuadro 1) y especies de las que $S$. nebouxii obtiene mate- 


\begin{tabular}{|c|c|c|c|c|c|}
\hline Especie & $\begin{array}{l}\text { Grado de } \\
\text { Utilización }{ }^{1}\end{array}$ & Fl & $\mathbf{F r}$ & $\mathbf{H}$ & $\mathbf{C}$ \\
\hline Bauhinia tomentosa $\mathrm{L}$. & Bajo & $\mathrm{X}$ & & & \\
\hline Ceiba speciosa (A. St.-Hil.) Ravenna & Bajo & $\mathrm{X}$ & & $\mathrm{X}^{\mathrm{t}}$ & $\mathrm{X}$ \\
\hline Dypsis lutescens (H. Wendl.) Beentje \& J. Dransf. & Bajo & & $\mathrm{X}$ & & \\
\hline Syzygium jambos (L.) Alston & Medio & & $\mathrm{X}$ & & \\
\hline Inga feuillei $\mathrm{DC}$. & Alto & & $\mathrm{X}$ & & \\
\hline Ficus bejamina $\mathrm{L}$. & Bajo & & $\mathrm{X}$ & & \\
\hline Hibiscus rosa-sinensis $\mathrm{L}$. & Medio & $\mathrm{X}$ & & $\mathrm{X}$ & \\
\hline Jacaranda mimosifolia D. Don & Bajo & & $\mathrm{X}$ & & \\
\hline Carya illinoinensis (Wangenh.) K. Koch & Alto & $\mathrm{X}$ & $\mathrm{X}$ & $\mathrm{X}^{\mathrm{t}}$ & $\mathrm{X}$ \\
\hline Juglans neotropica Diels & Alto & & $\mathrm{X}$ & & \\
\hline Melia azedarach $\mathrm{L}$. & Medio & & $\mathrm{X}$ & & \\
\hline Morus nigra $\mathrm{L}$. & Medio & & $\mathrm{X}$ & $\mathrm{X}$ & \\
\hline Phoenix spp. & Alto & $\mathrm{X}$ & $\mathrm{X}$ & & \\
\hline Prunus spp. & Medio & & $\mathrm{X}$ & & \\
\hline Malus domestica (Suckow) Borkh. & Medio & & $\mathrm{X}$ & & \\
\hline Spathodea campanulata P. Beauv & Medio & & $\mathrm{X}$ & & \\
\hline Tipuana tipu (Benth.) Kuntze & Alto & $\mathrm{X}$ & $\mathrm{X}$ & & $\mathrm{X}$ \\
\hline
\end{tabular}

Obtenido de apuntes de observaciones directas de los individuos alimentándose o a partir de rastros en las 20+ visitas realizadas al área de estudio previas a la recolección de datos para el presente estudio.

${ }^{1}$ Grado de Utilización: Alto = Observado en todas las visitas al área; Medio = observado en al menos cinco de visitas al área; Bajo = observado en no más de tres ocasiones. Partes utilizadas: Flor $(\mathrm{Fl})$, Fruto $(\mathrm{Fr})$, Hojas (H, el superíndice t señala el consumo de hojas tiernas o brotes), Corteza (C).

Cuadro 1. Especies utilizadas como alimento por S. nebouxii en el campus de la Pontificia Universidad Católica del Perú.

riales para la construcción de su madriguera en el campus de la PUCP (Cuadro 2) fueron determinadas con base en observaciones directas realizadas durante evaluaciones preliminares entre los años 2012 y 2013, respectivamente.

Se hallaron los parámetros más importantes para cada variable numérica evaluada e histogramas de frecuencia para los rangos de valores seleccionados según el caso; mientras que para variables cualitativas se hizo únicamente análisis a partir de los histogramas. Adicionalmente se buscó correlaciones utilizando rangos de
Spearman entre los pares de variables de área circundantes (DEN, NAA, NAC, NAM, COD, PAE), correlación por coeficiente de Pearson entre las variables de estructura de los arboles con madriguera (ATA, DAP, CCA, AMC), entre las alturas del árbol y la madriguera (ATA-ALM) y alturas mínima de copa y de madriguera (AMC-ALM). Además se hizo un análisis de la altura relativa de la madriguera (ARM), hallada con la proporción altura de la madriguera y altura del árbol (ALM/ATA) y se ordenó por frecuencia de clases, ya que algunos autores han señalado que la altura de 


\begin{tabular}{llll}
\hline Especie & H & R & O \\
\hline Acacia retinodes Schltdl. & $\mathrm{X}$ & & \\
Cedrela odorata $\mathrm{L}$. & $\mathrm{X}$ & & \\
Ceiba sp. & $\mathrm{X}$ & & $\mathrm{X}^{\mathrm{b}}$ \\
Eucalyptus camaldulensis Dehnh. & $\mathrm{X}$ & $\mathrm{X}$ & \\
Inga feuillei DC. & $\mathrm{X}$ & & \\
Ficus benjamina $\mathrm{L}$. & $\mathrm{X}$ & $\mathrm{X}$ & \\
Fraxinus sp. & $\mathrm{X}$ & $\mathrm{X}$ & $\mathrm{X}^{\mathrm{a}}$ \\
Harpullia arbórea Radlk. & $\mathrm{X}$ & & \\
Melia azedarach L. & $\mathrm{X}$ & & \\
Parkinsonia aculeata L. & $\mathrm{X}$ & $\mathrm{X}$ & \\
Phoenix sp. & $\mathrm{X}$ & & $\mathrm{X}^{\mathrm{c}}$ \\
Schinus molle L. & $\mathrm{X}$ & & \\
Spathodea campanulata P. Beauv & $\mathrm{X}$ & & \\
Tipuana tipu (Benth.) Kuntze & $\mathrm{X}$ & & \\
\hline
\end{tabular}

Partes utilizadas: hojas $(\mathrm{H})$, pequeñas ramas $(\mathrm{R}) \mathrm{u}$ otros $(\mathrm{O}$, el superíndice indica a $=$ racimos de semillas, $\mathrm{b}=$ fibras del fruto $\mathrm{y} \mathrm{c}=$ fibras del estípite) .

Cuadro 2. Especies utilizadas por S. nebouxii para la construcción de sus madrigueras en el campus de la Pontificia Universidad Católica del Perú.

la madriguera depende de la altura del árbol que la sostiene (Brown y McGuire 1975; Tittensor 1970; Williams 2011). Finalmente, se realizó una prueba de chi-cuadrado de bondad de ajuste para una distribución uniforme con la altura relativa de la madriguera en la copa del árbol (ARM); y un análisis de la posición relativa de la misma por medio de un gráfico bidimensional (scatterplot XY).

\section{Resultados}

En total se registraron 33 madrigueras distribuidas en 25 árboles del campus, lo que corresponde a una densidad de dos madrigueras/ hectárea (de área verde). En su mayoría se encontraban en áreas aledañas a la vereda principal del campus.

\section{(A) Áreas circundantes}

La evaluación mostró que la cobertura de dosel (COD) es la variable con menor dispersión $(\mathrm{CV}=0.21)$, seguida de densidad de árboles $(\mathrm{DEN}, \mathrm{CV}=0.43)$ (Cuadro 3$)$. En cuanto a las frecuencias, para la variable densidad de árboles (DEN) se halló mayores registros en el rango 21-35 arb/ha (Figura 1a), y aunque el promedio está un poco encima de dichos valores (37.48 $\pm 6.39 \mathrm{arb} / \mathrm{ha})$, en general se encontraron densidades por encima de 20 y hasta 70 árboles/ha. Además, se encontraron hasta 3 árboles adicionales con 1 o más madrigueras (NAA) por área evaluada, siendo lo más frecuente un único árbol adicional con madriguera(s) (Figura 1b). La distribución de la variable relacionada al alimento (NAC) mostró 


\begin{tabular}{ccccc}
\hline \multirow{2}{*}{ Variable } & $\begin{array}{c}\text { Promedio } \\
\boldsymbol{\mu} \pm \mathbf{I C}(\mathbf{9 5 \%})\end{array}$ & Rango & $\begin{array}{c}\text { Desv. Est } \\
\boldsymbol{\sigma}\end{array}$ & $\mathbf{C V}$ \\
\hline DEN (arb/ha) & $37.48 \pm 6.39$ & $15.2-71.3$ & 16.29 & 0.43 \\
NAA & $1.2 \pm 0.29$ & $0-3$ & 0.75 & 0.62 \\
NAC & $2.96 \pm 0.86$ & $0-6$ & 2.18 & 0.74 \\
NAM & $2.84^{*}$ & $0-6$ & 1.95 & 0.69 \\
COD (\%) & $50.83 \pm 4.17$ & $33.3-75$ & 10.64 & 0.21 \\
\hline
\end{tabular}

Densidad de árboles (DEN), Número de árboles adicionales con madriguera (NAA), Número de árboles o arbustos con alguna parte que constituya alimento para la especie (NAC), Número de árboles o arbustos con materiales para la construcción de la madriguera (NAM), Cobertura de dosel (COD). El asterisco denota datos que no presentan distribución normal, por lo que no se considera el IC (95\%).

Cuadro 3. Parámetros estadísticos hallados para las variables de áreas circundantes a los árboles con madriguera $(\mathrm{N}=25)$.
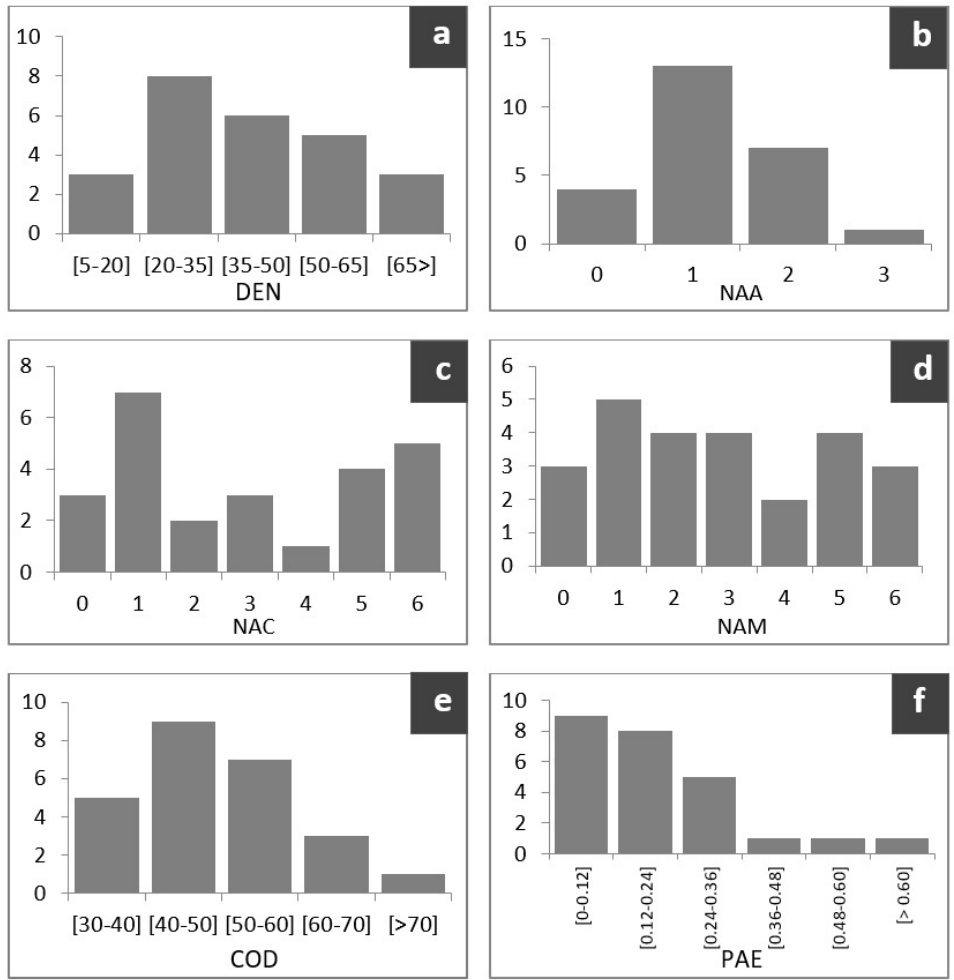

Figura 1. Histogramas de frecuencia de las variables de caracterización de áreas circundantes a la madriguera $(\mathrm{N}=25)$. a: Densidad (DEN) en árboles por hectárea, b: Número de árboles adicionales con madriguera (NAA), c: Número de árboles o arbustos con alguna parte que constituya alimento (NAC), d: Número de árboles con materiales para la construcción de madrigueras (NAM), e: Cobertura de dosel (COD) en porcentaje, f: Proporción de área edificada (PAE). 
un patrón irregular, sin embargo, se pudo ver que un árbol o arbusto con alimento resultó ser el valor más frecuente y las parcelas con 5 y 6 árboles o arbustos fueron también frecuentes (Figura 1c); asimismo, el promedio fue de 3 individuos $(\sigma=2.18)$. En cuanto a los árboles con materiales para la construcción de madrigueras (NAM) se encontró que la ausencia de éstos resultó ser mucho menos frecuente que la presencia de 1 o más árboles, como máximo se encontraron 6 árboles dentro de las áreas analizadas (Figura 1d). Por su parte, la mayor frecuencia de cobertura de dosel (COD) fue de alrededor del 50\% en las áreas circundantes a los árboles con madriguera (Figura 1e), lo que coincidió con el promedio que fue de $50.8 \%$. Aunque la mayoría de madrigueras se encuentran ubicadas cerca de edificaciones, no son las áreas edificadas las que dominan el área circundante a las madrigueras, ya que el $88 \%$ de las áreas evaluadas no presentaron más de 0.4 en la proporción área edificada/área total (PAE, Figura 1f). Finalmente, para esta escala, los análisis de correlación por rangos de Spearman mostraron 2 correlaciones significativas entre pares de variables, estas fueron NACNAM $(\rho p=0.988)$ y NAA-COD $(\rho p=0.630)$.

\section{(B) Árboles con madriguera}

Las madrigueras de Simosciurus nebouxii se encontraron en árboles de 6 especies, agrupando 2 especies del género Fraxinus por ser casi idénticos en estructura (Figura 2). El 44\% del total de árboles con madriguera se trató de Tipuana tipu (Benth.) Kuntze, siendo Fraxinus sp. (20\%) y Eucalyptus camaldulensis Dehnh. (20\%) las especies con frecuencias relativamente altas también. El resto de las especies estuvieron escasamente representadas. El número de madrigueras encontradas por árbol (NMA) para el periodo en evaluación fue de 1 a 4; 20 de los arboles (80\%) presentaban una única madriguera, mientras que árboles con 2 , 3 y 4 madrigueras fueron mucho menos frecuentes (Cuadro 4).

En cuanto a las variables de estructura (Figura 3), las clases más frecuentes fueron $30-40 \mathrm{~cm}$ de diámetro a la altura del pecho (DAP), 12.5-

\begin{tabular}{ccc}
\hline $\begin{array}{c}\text { Número de } \\
\text { madrigueras }\end{array}$ & Frecuencia & Porcentaje \\
\hline 1 & 20 & 80 \\
2 & 3 & 12 \\
3 & 1 & 4 \\
4 & 1 & 4 \\
Total & 25 & 100 \\
\hline
\end{tabular}

Cuadro 4. Frecuencia y porcentaje de número de madrigueras presentes por árbol encontradas en el campus de la Pontificia Universidad Católica del Perú.

14 metros de altura total (ATA), 5-6 m de altura mínima de copa (AMC), 0-50 y 140-160 m² de amplitud de copa (ACA) presentando una distribución bimodal $\mathrm{y}$, finalmente, $60-70 \%$ de cobertura de copa (CCA). Así también, las variables de estructura que presentaron menor variabilidad fueron altura total (ATA) y cobertura de copa (CCA), mientras que las variables con los valores más dispersos fue amplitud de la copa (ACA) y el diámetro a la altura de pecho (DAP, Cuadro 5).

Finalmente, el valor del coeficiente de correlación de Pearson entre el par de variables DAPACA, fue el único valor significativo entre los pares de variables analizados, con $\rho p=0.522$ $(p$-valor $=0.007)$.

\section{(C) Posición de la madriguera en el árbol}

De las 33 madrigueras encontradas, 27 se encontraron en las ramas de los árboles y 6 pegadas al tronco principal o sobre una bifurcación del mismo. Las variables evaluadas a esta escala muestran una alta variabilidad de la posición horizontal de la madriguera dentro de la copa, teniendo valores que varían de 0 a $6.3 \mathrm{~m}(\mathrm{CV}=$ 0.69) en distancia al eje del árbol (DEA), y de 0.3 a $5.8 \mathrm{~m}(\mathrm{CV}=0.55)$ en distancia al borde de la copa (DBC). Mientras que la variabilidad de la altura a la que se encuentra (ALM) es mucho menor $(\mathrm{CV}=0.29)$, a pesar de mostrar un rango de valores que parece ser más amplio (Cuadro 6). De manera más detallada, se encontró que la altura de las madrigueras (ALM) en promedio fue de $9.13 \mathrm{~m}(\sigma=2.71)$, variando en un rango de más de 10 metros de diferencia; 


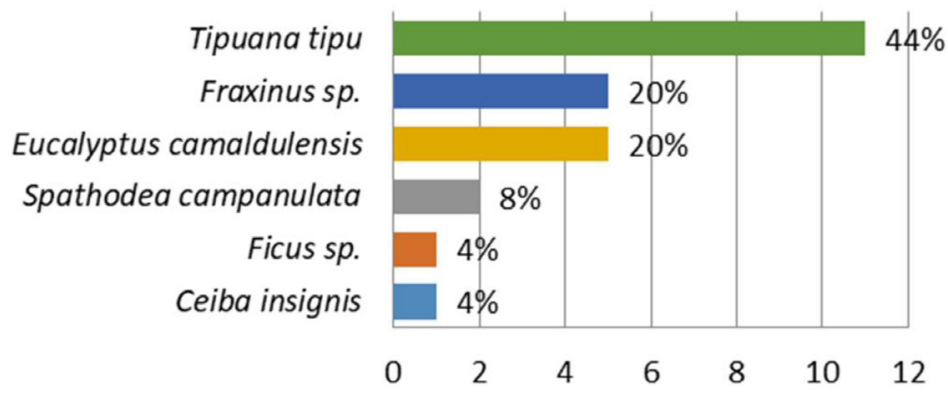

Figura 2. Frecuencia y porcentaje de árboles con madriguera(s) por especie encontrados en el campus de la Pontifica Universidad Católica del Perú (N=25).


Figura 3. Histogramas de frecuencia de las variables de estructura de los árboles con madrigueras $(\mathrm{N}$ = 25). a: Diámetro a la altura de pecho (DAP) en centímetros, b:Altura total (ATA) en metros, c: Altura mínima de copa (AMC) en metros, d: Amplitud de copa (ACA) en metros cuadrados, e: Cobertura de copa (CCA) en porcentaje. 


\begin{tabular}{lcccc}
\hline \multicolumn{1}{c}{ Variable } & $\begin{array}{c}\text { Promedio } \\
\boldsymbol{\mu} \pm \mathbf{I C}(\mathbf{9 5 \%})\end{array}$ & Rango & $\begin{array}{c}\text { Desv. Est } \\
\boldsymbol{\sigma}\end{array}$ & $\mathbf{C V}$ \\
\hline DAP $(\mathrm{cm})$ & $47.6 \pm 5.8$ & $21.0-77.9$ & 14.8 & 0.31 \\
ATA $(\mathrm{m})$ & $11.8 \pm 0.8$ & $8.7-15.6$ & 2.2 & 0.18 \\
AMC $(\mathrm{m})$ & $5.7 \pm 0.4$ & $3.7-8.6$ & 1.3 & 0.22 \\
ACA $\left(\mathrm{m}^{2}\right)$ & $94.2^{*}$ & $21.9-187.3$ & 54.5 & 0.58 \\
CCA $(\%)$ & $67.7 \pm 5.1$ & $35.0-92.0$ & 12.9 & 0.19 \\
\hline
\end{tabular}

Diámetro a la altura de pecho (DAP), Altura total (ATA), Altura mínima de copa (AMC), Amplitud de copa (ACA) y Cobertura de copa (CCA), El asterisco indica que los datos no presentaron distribución normal por lo que no se considera el IC (95\%).

Cuadro 5. Parámetros estadísticos de variables de estructura de árboles con madriguera ( $\mathrm{N}=25)$.

\begin{tabular}{ccccc}
\hline Variable & $\begin{array}{c}\text { Promedio } \\
\boldsymbol{\mu} \pm \mathbf{I C} \\
\mathbf{( 9 5 \% )}\end{array}$ & Rango & $\begin{array}{c}\text { Desv. Est. } \\
\boldsymbol{\sigma}\end{array}$ & $\mathbf{C V}$ \\
\hline ALM (m) & $9.1 \pm 0.93$ & $4.7-14.9$ & 2.71 & 0.29 \\
DEA (m) & $2.6 \pm 0.61$ & $0.0-6.3$ & 1.79 & 0.69 \\
DBC (m) & $2.5 \pm 0.47$ & $0.3-5.8$ & 1.37 & 0.55 \\
\hline
\end{tabular}

Altura (ALM), Distancia al eje del árbol (DEA) y Distancia al borde de la copa (DBC).

Cuadro 6. Parámetros estadísticos de posición de madriguera en el árbol $(\mathrm{N}=33)$.

con el $60.6 \%$ de las madrigueras en el rango de 7-11 metros y las restantes distribuidas de manera más uniforme en los rangos de clase restantes (Figura 4a). La posición horizontal de la madriguera fue muy variable, presentándose en ocasiones sobre el mismo tronco o muy cerca del borde de la copa. La variable distancia de la madriguera al eje del árbol (DEA) presentó una distribución bimodal (Figura 4b), con 1 y 4 metros como distancias más frecuentes, mientras que los menos frecuentes fueron los valores extremos. Un caso diferente fue el de la distancia de la madriguera al borde de la copa (DBC, Figura 4c), variable que presentó mayor frecuencia en los valores más pequeños, es decir, los más cercanos al borde de la copa; mientras que los valores más altos presentaron mucha menor frecuencia. Los valores encontrados para estas dos variables (DEA y DBC) estuvieron dentro de los rangos [0-6.3], y [0.35.8] metros, respectivamente. El análisis de la altura relativa de la madriguera (ARM) mostró un promedio de $0.77(\sigma=0.14$; rango [0.45$0.97])$, y una mayor frecuencia de valores entre 0.8 y 0.9 (Figura 4d). Además, a partir del análisis de la gráfica de frecuencias de la orientación cardinal en 12 categorías (Figura 5) se pudo ver que muestra una distribución aleatoria, aunque el bajo número de observaciones impide realizar un análisis estadístico para determinar bondad de ajuste a una distribución determinada.

A partir del análisis de correlación de Pearson entre la altura total del árbol y la altura de la madriguera (ATA-ALM) se halló una correlación positiva de $\rho p=0.679$ ( $p$-valor $<$ 0.01 ). De modo similar, el análisis de correlación de Pearson entre altura mínima de copa y altura de madriguera (AMC-ALM) arrojó una correlación positiva moderada $(\rho p=0.546$, $p$-valor $=0.001)$. En el caso de otros pares de 

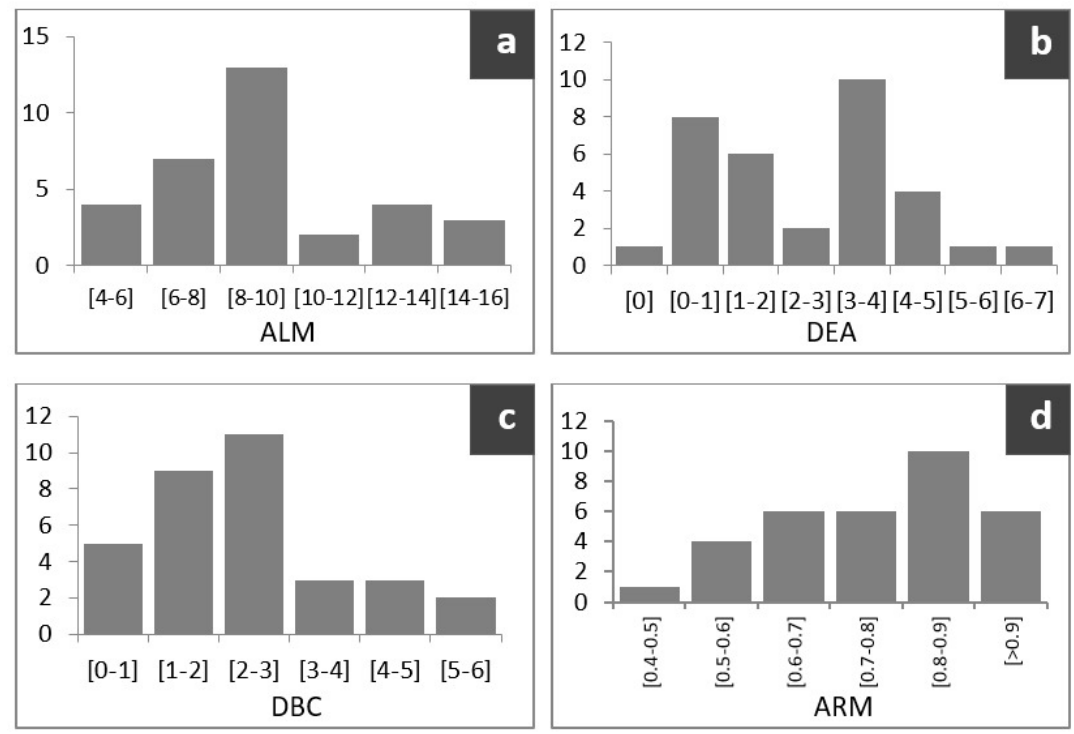

Figura 4. Histogramas de frecuencia de las variables de posición de la madriguera $(\mathrm{N}=33)$. a: Altura (ALM) en metros, b: Distancia al eje del árbol (DEA) en metros, c: Distancia al borde de la copa (DBC) en metros, d: Altura relativa de la madriguera (ARM).

variables no se encontró correlaciones con significancia.

La prueba de bondad de ajuste de la altura relativa de la madriguera en la copa del árbol (ARM) para una distribución uniforme no mostró diferencias, siendo el número de madrigueras similar en la parte alta $(\mathrm{n}=14)$, media $(\mathrm{n}=10)$ y baja $\left(\mathrm{n}=9 ; \mathrm{X}^{2}=1.27\right.$, g.l. $=2, p=$ $0.529)$ de la copa del árbol.

Finalmente, al analizar de forma gráfica la posición relativa de las madrigueras en la copa del árbol (Figura 6), sin considerar la dirección cardinal, se pudo observar cómo las madrigueras se ordenan en el área de lo que podría ser el perfil de la copa de un árbol (con el eje Y como eje central con valores correspondientes a la posición de la madriguera entre la altura mínima de copa y la altura total del árbol, y X como la posición horizontal de la madriguera respecto al eje central y el borde de la copa). En cuanto a la ubicación de los nidos por especie, las madrigueras en Fraxinus se encontraron en la mitad inferior de la copa; mientras que en Tipuana hubo un mayor número de madri- gueras en la parte superior, pero los números analizados no son tan altos para asegurar que existen tendencias marcadas.

\section{Discusión}

Las madrigueras estaban distribuidas en su mayoría a zonas aledañas a la vereda principal del campus. Al respecto, Salsbury (2008), quien realizó un estudio en las madrigueras de Sciurus niger Linnaeus en Indiana, EUA, reportó que la distribución no estaba influenciada por variables de atributos de los parches de vegetación en los que se encuentran, tales como extensión del parche o aislamiento, lo cual corresponde a lo encontrado, ya que la vereda principal es muy transitada por lo que el aislamiento del área no sería un atributo importante para la construcción de madrigueras.

\section{(A) Áreas circundantes}

Haciendo una comparación entre los datos de densidad de árboles encontrados en el presente estudio y las de áreas de bosque donde la ardilla de nuca blanca se distribuye natural- 




Figura 5. Orientación cardinal de la madriguera respecto al eje del árbol.

mente, se puede ver que corresponde a valores similares, como es el caso del Santuario Nacional Bosque de Pómac en Lambayeque, donde se reporta que la densidad de árboles con DAP $\geq 45 \mathrm{~cm}$ es de entre 16 y 39 árboles/ha (SERNANP 2010). Aunque el área evaluada se trata de una zona urbana, en que la distribución y densidad de árboles es artificial, es posible que cumpla el requerimiento de cobertura reproductiva en este aspecto.

$\mathrm{Al}$ respecto de la variable NAA, en la que se encontró un único árbol adicional con madriguera como valor más frecuente, se sabe que las ardillas arborícolas, como Siciurus carolinensis Gmelin, pueden construir y utilizar más de una madriguera, ubicándose éstas a distancias cortas unas de otras (Shorten 1951). Pero cuando se trata de madrigueras pertenecientes a diferentes individuos suelen encontrarse de manera espaciada debido a un aumento en la agresividad de las hembras durante la época de cría (Gurnell 1987). Se desconoce si en el caso de $S$. nebouxii en su hábitat natural ocurre lo mismo, y aun cuando fuera así, las condiciones existentes en el hábitat urbano podrían provocar que exista cercanía entre madrigueras de distintos individuos debido a un cambio en el comportamiento como producto de la adaptación a condiciones de limitada extensión de cobertura reproductiva (Parker y Nilon 2008), por lo que no se puede asumir que las madrigueras encontradas en el mismo árbol pertenecieran a un solo individuo.

En cuanto a la variable de disponibilidad de alimento (NAC), estudios de especies en el hemisferio norte han mostrado que una alta densidad de árboles productores de semillas o la cercanía a agrupamientos de estas especies (coníferas, por ejemplo) son importantes para el establecimiento de madrigueras en dichos lugares, y que de faltar especies preferidas en el área otros recursos seleccionados de forma secundaria pasan a ser más utilizados en determinados hábitats (Valhe y Patton 1983; Williams 2011). Puede ser éste también el caso de $S$. nebouxii, ya que se ha encontrado que 13 de las 25 áreas evaluadas (52\%) tienen 3 o más árboles o arbustos que se consideran favoritos, y 9 de ellas (36\% del total) tiene 6 árboles o arbustos que brindan alimentos.

Respecto a los resultados de la variable relacionada con los materiales para construcción de madrigueras (NAM), algunos autores señalan que las ardillas arborícolas, en la mayoría de los casos, utilizan material tomado de los mismos árboles donde construyen las madrigueras. Pero este material solamente es utilizado para la parte exterior de sus madrigueras; el recubrimiento interno está constituido de materiales de textura suave y dichos materiales parecen ser buscados con mayor cuidado, prefiriendo ciertas especies de vegetación y rechazando otras, aun cuando sean muy abundantes dentro de su home-range (Brown y McGuire 1975; Raspopov e Isakov 1980). Es por ello que los hallazgos presentados son una primera referencia para el estudio de este aspecto de la especie, siendo recomendable en futuras investigaciones incluir la procedencia de los materiales utilizados para la construcción de las madrigueras, describiendo a qué especie corresponden las ramas, fibras y otros materiales vegetales utilizados.

Por otro lado, el $84 \%$ de madrigueras se encuentran en áreas con coberturas de entre 30 y $60 \%$. En la literatura existen reportes de preferencia de algunas especies de ardillas por 


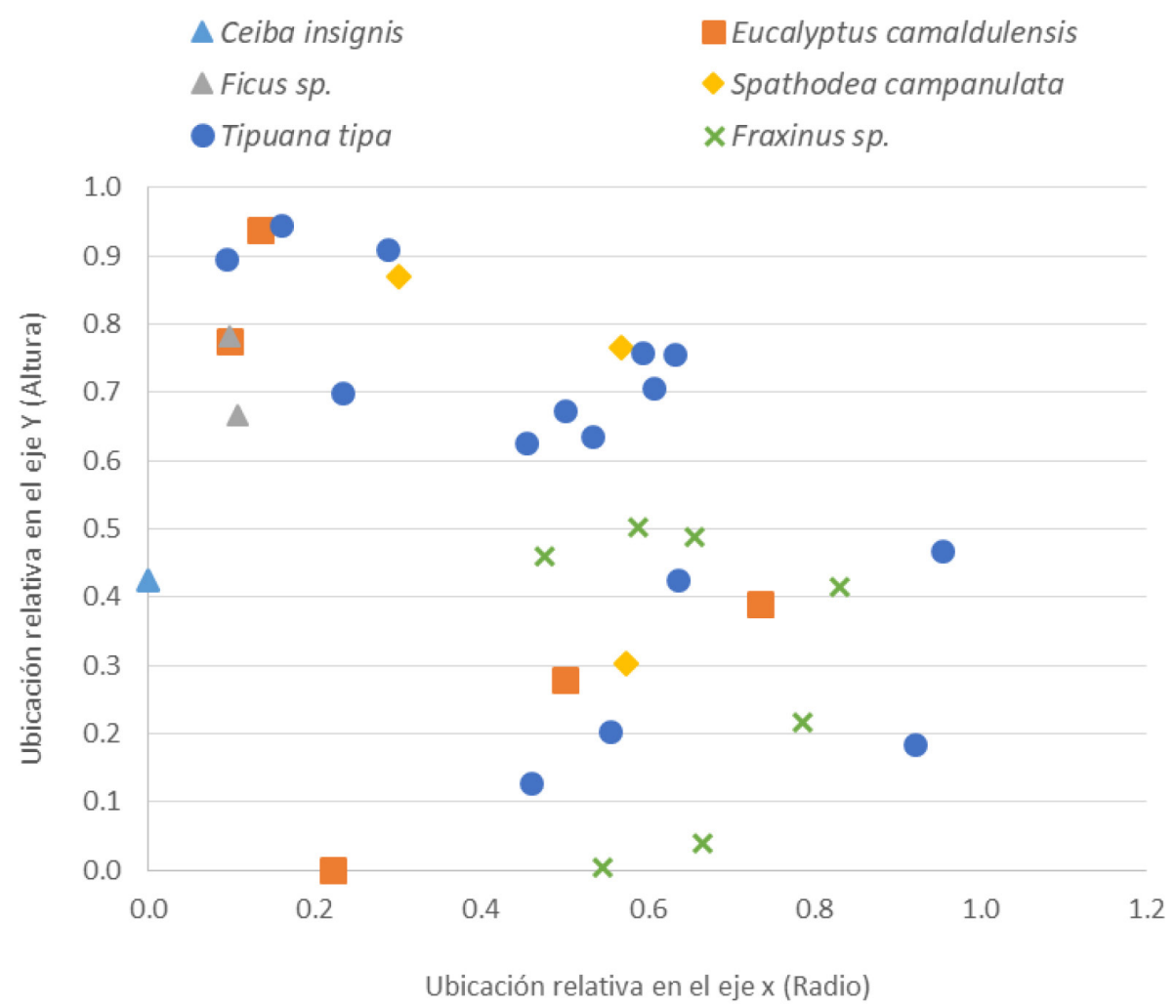

Figura 6. Posición relativa de las madrigueras en la copa del árbol por especie.

ciertos valores de cobertura, como es el caso de Sciurus aberti Woodhouse, especie que prefiere áreas con mayor cobertura que la especie simpátrica Tamiasciurus hudsonicus Erxleben (Edelman et al. 2009). Y, en general para las ardillas arborícolas, se han encontrado valores de cobertura por encima de la media en las áreas elegidas para la construcción de madriguera respecto a otras áreas de los bosques evaluados (Edelman y Koprowski 2005; Merrick et al. 2007; Williams 2011). Es necesario mencionar también que se trata de una variable estacional debido a la presencia de especies caducifolias tanto oriundas como foráneas, alternando periodos de pérdida de hojas a lo largo del año, por lo que sería necesario realizar evaluaciones en las diferentes estaciones para contrastar resultados.
La influencia del carácter antrópico del área es relevante para el estudio de la variable PAE. Como se pudo constatar a partir de las imágenes satelitales y las visitas al área de estudio, son las áreas con más edificaciones las que poseían en sus cercanías los árboles de mayor antigüedad y por ende de mayor tamaño y cobertura, mientras que las áreas abiertas poseían arboles más jóvenes. Aunque los datos disponibles son insuficientes para sacar conclusiones, la evidencia sugiere que son las características de los árboles las más relevantes para la selección de los sitios para la construcción de madrigueras, aun por encima de las desventajas que podrían presentar áreas transitadas o áreas con presencia de edificaciones. Además, varios autores firman que la cobertura, natural o artificial, lejos de constituir un inconve- 
niente, puede convertirse en un recurso más cuando las ardillas arborícolas la encuentran adecuada como estructura de sostén o de protección para sus madrigueras (Middelton 1931, Shorten 1962, citados por Tittensor 1970).

La correlación del par de variables NACNAM se explica, debido a que las especies que brindan alimento también son usadas para extraer materiales. Mientras que la relación NAA-COD sugiere que las áreas con mayores valores de cobertura también son las que poseen más madrigueras, siendo recomendable análisis más profundos para determinar con precisión la importancia de la variable cobertura en la selección del lugar para la construcción de la madriguera.

\section{(B) Árboles con madriguera}

El hecho de que tres especies de árboles, de las más de 40 de que había en el campus de la PUCP en el momento de la evaluación, agrupen el $84 \%$ de los árboles con madrigueras coincide con lo previamente reportado para otras especies de ardillas arborícolas, las que suelen tener preferencia por algunas especies de árboles, al mismo tiempo que evitan otras. Por ejemplo, Sciurus carolinensis, una especie introducida en Reino Unido, utiliza preferentemente árboles de roble (Quercus robur L.) para la construcción de sus madrigueras, al mismo tiempo que evita el fresno (Fraxinus excelsior L.), aun cuando ambas| especies de árboles son muy abundantes; además difieren de las preferidas por la especie nativa Sciurus vulgaris Linnaeus, que prefiere las coníferas (Shorten 1951; Tittensor 1970).

La predominancia de una única madriguera por árbol (NMA) en los árboles que las presentaban coinciden con los reportes para otras especies como Sciurus vulgaris (Tittensor 1970), Sciurus carolinensis (Sanderson et al. 1976), Sciurus aberti (Edelman y Koprowski 2005) y Sciurus niger (Salsbury 2008). Williams (2011), encontró que el número de madrigueras de Sciurus carolinensis presentes en cada árbol estaba relacionado a la densidad arbórea y al DAP, pero también a la cercanía al alimento, representado por una especie en par- ticular (Cliftonia sp.). Por otro lado, en este estudio, los árboles que presentaron más de una madriguera pertenecían a diferentes especies (Tipuana tipu, Ficus sp., Faxinus americana y Spathodea campanulata P. Beauv.), lo que puede indicar que la especie de árbol no influencia el comportamiento de la variable. De tal modo, el comportamiento de la variable NMA puede estar más relacionada a la disponibilidad de recursos (cobertura y alimento) y no a la especie de árbol donde se encuentran.

En cuanto a las variables de estructura, la altura total de los árboles (ATA) está relacionada con el DAP, por lo que se esperaría que los coeficientes de variación no sean tan distintos, sin embargo, el hecho de que la altura total tenga un coeficiente de variación pequeño en comparación con el DAP se puede deber al manejo de los árboles, los cuales son podados con cierta frecuencia alterando la relación altura-DAP. Por otro lado, la alta variabilidad de la amplitud de copa (ACA) se debería a las diferencias de estructura de las distintas especies, algunas con copas que al crecer se extienden horizontalmente (como Tipuana) y otras que más bien tienen una copa compacta con ramas que crecen hacia arriba (como en el caso Spathodea).

Finalmente, la correlación encontrada entre DAP y ACA no muestra tendencias importantes con relación a la ubicación de las madrigueras dado que ambas variables usualmente están correlacionadas. Por otro lado, la correlación positiva que se esperaría para el DAP y la altura total (ATA) no se encontró, lo cual, como ya se mencionó, puede deberse al manejo constante de los árboles del campus por medio de podas.

\section{(C) Posición de la madriguera en el árbol}

La alta variabilidad en la posición horizontal de la madriguera dentro de la copa del árbol indicaría que dichas variables no son determinantes en la elección de la posición final de la madriguera, como sí lo sería la variable altura (ALM), dado que posee un coeficiente de variación mucho menor. Esta aparente preferencia por una altura va de acuerdo con lo reportado por varios autores. Las ardillas ar- 
borícolas construyen sus nidos raramente por debajo de los 3 metros y a veces alcanzando hasta 25 metros (Middleton 1931, Allen 1943, Baumgartner 1943, Layne 1954, citados por Tittensor 1970), rango que se vuelve más estrecho cuando se trata de una sola especie y que se hace evidente cuando varias especies se presentan en simpatría (Edwards y Guynn 1995).

Del mismo modo, el análisis adicional de los valores de ARM (proporción ALM/ATA) en los que se encontró un valor promedio de 0.77 \pm 0.05 , resultan ser similares a lo reportado para $S$. aberti en diferentes estudios. Para dicha especie se ha encontrado que la madriguera estaba construida aproximadamente a un 0.75 de la altura del árbol, como lo reportado por Farentinos (1972) quien encontró que la altura relativa estaba dentro del rango $0.63-0.98$, mientras que Snyder y Linhart (1994), Halloran y Bekkof (1994) y Edelman y Koprowski (2005), reportan los siguientes promedios: 0.68 \pm 0.04 , de $0.72 \pm 0.11$ y $0.733 \pm 0.012$.

Por otro lado, los rangos amplios de las variables DEA y DBC sugieren que ninguna de las dos variables es de gran importancia en la selección del sitio para la construcción de la madriguera. Aunque la bibliografía al respecto es escasa, se pueden señalar los estudios de Williams (2011) y de Edelman y Koprowski (2005). En el primero se reportó para Sciurus carolinensis, en un bosque mixto, que la posición de las madrigueras iba desde el tronco principal hasta los extremos de las ramas, sin preferencia aparente alguna; mientras que en el segundo se encontró que Sciurus aberti, en un bosque mixto de coníferas, construyó madrigueras junto al tronco principal en su mayoría. Por esto hacen falta más datos para determinar si la posición horizontal está relacionada a la arquitectura de los árboles utilizados o si se trata de preferencias inherentes a la especie.

La distribución aleatoria de la orientación cardinal de madriguera en el eje del árbol (Figura 5) contrasta con lo encontrado en la literatura sobre otras especies. En el hemisferio norte, en zonas más alejadas del ecuador y donde la luz solar es importante para la termorregulación en algunas épocas del año, se ha encontrado mayor frecuencia en orientaciones hacia el sur y el este; y aunque la ubicación no está restringida para estas direcciones se explica debido a las mayores cantidades de radiación solar y los primeros rayos solares al amanecer, respectivamente. Este es el caso de Sciurus aberti en Colorado y Arizona (Farentinos 1972; Snyder y Linhart 1994; Edelman y Koprowski 2005). Sin embargo no se trata de un comportamiento común para todas las especies. Ramos-Lara y Cervantes (2007) en un estudio llevado a cabo en Michoacán, México, con Sciurus aureogaster Cuvier, encontraron que los nidos estaban orientados aleatoriamente respecto al eje del árbol; lo mismo fue reportado por Cudworth y Koprowski (2011) para Sciurus arizonensis Coues en Arizona, EUA. De tal modo, aunque los resultados encontrados para Sciurus nebouxii en esta locación podrían deberse a que el fotoperiodo, albedo y otras variables climáticas no varían en gran medida a lo largo del año, no es posible asegurarlo solamente con los datos obtenidos en este estudio, y sería recomendable medir más variables relacionadas a la termorregulación y la influencia de las condiciones climáticas en el comportamiento de la especie respecto a esta variable.

La correlación encontrada en ATA-ALM coincide con otros estudios como los reportados para la especie Sciurus aeurogaster por Brown y McGuire (1975) y Ramos-Lara y Cervantes (2007), en áreas donde la especie fue introducida y donde es nativa, respectivamente. Mientras que la correlación AMC-ALM no ha sido estudiada previamente, por lo que estos datos pueden servir para comparaciones futuras, pudiendo contribuir a determinar si la altura de la madriguera se elige únicamente en función a la preferencia por parte de la especie o si está relacionada a algún atributo del árbol que pudiera conferir alguna ventaja como mayor protección contra el viento, menor visibilidad desde el aire o el suelo, etc.

La distribución uniforme de madrigueras en la copa es similar a lo encontrado en Sciurus aureogaster (Ramos-Lara y Cervantes 2007). En contraste, para otras especies como Sciurus aberti y Sciurus arizonensis se ha reportado 
mayores frecuencias en el tercio más alto de la copa del árbol (Brown y Macguire 1975; Halloran y Bekkof 1994; Cudworth y Koprowski 2011).

Finalmente, las tendencias observadas en la posición relativa de las madrigueras en la copa del árbol son difíciles de analizar debido al reducido tamaño de la población en estudio. Sin embargo, la Figura 6 muestra como la posición relativa de las madrigueras sigue la forma de la copa de un árbol promedio, encontrándose en su mayoría justo por debajo del límite de la copa del árbol. Respecto a este último punto Cudwoth y Koprowski (2011) afirman que las partes más altas de los árboles y adyacentes al tronco proveen cobertura foliar densa, mayor estabilidad al nido y protección del viento y elementos. No obstante, las posiciones de las madrigueras encontradas en el presente estudio están distribuidas de manera bastante uniforme. Esta diferencia se puede deber a la arquitectura de las especies arbóreas predominantes en el campus de la PUCP, la misma que resulta muy diferente de las encontradas en coníferas las cuales son más comunes en Norteamérica, donde se han hecho la mayor parte de estudios de esta naturaleza para especies similares.

\section{Conclusiones}

Los sitios utilizados para la construcción de los nidos fueron en su mayoría las áreas adyacentes a la vereda principal del campus. Dichas áreas tuvieron una densidad de árboles considerable para tratarse de un área urbana (37.5 árboles/ha en promedio) y además presentaron cobertura de media a alta (de 33 a 75\%). Asimismo, en la mayoría de los casos existía presencia de edificaciones en un radio de $25 \mathrm{~m}$, que en general ocupaban más del $35 \%$ de la superficie. Por otro lado, la aparente preferencia por unas pocas especies de árboles (géneros Tipuana, Fraxinus y Eucalyptus), altura (de 8.7 a $15.6 \mathrm{~m}$ ) y porcentaje de cobertura (de 35 a 92\%), como es de esperarse, sugieren coincidencias con otras especies de la misma familia de las que se tiene información. Respecto a la posición del nido en la copa del árbol, se encontró una fre- cuencia alta en el rango de 8 a $10 \mathrm{~m}$ de altura, y una posición horizontal muy variable, al igual que la dirección cardinal respecto al tronco. Sin embargo se puede identificar un patrón en la distribución de las madrigueras que las ordena justo por debajo del borde del borde del follaje de la copa, siguiendo la forma de la misma. En conclusión, se sugiere que las variables más importantes en la descripción de la ubicación de la madriguera son la cobertura de dosel del área donde se ubica el árbol que sostiene la madriguera (COD), la especie y la cobertura de dicho árbol (CCA) y la altura a la que se ubica la madriguera (ALM). Sin embargo, es necesario continuar con estudios relacionados al tema para poder describir con mayor claridad la cobertura vegetal que utiliza la especie Simosciurus nebouxii y cómo la utiliza.

\section{Agradecimientos}

Este trabajo fue posible gracias a Sarah Landeo, Manuel Quispe, Joseph Herrera y David Aybar quienes prestaron su apoyo en la colecta de datos; a Daniel Montes quien proporcionó valiosa información; a Fernando Roca quien otorgó el permiso para el estudio en del campus de la Pontificia Universidad Católica del Perú; a Carlos Sotomayor y Amparo Gómez de la Oficina de Mantenimiento del campus quienes brindaron apoyo en la logística.

\section{Bibliografía}

Allen, DL. 1943. Michigan fox squirrel management. Michigan, USA. Michigan Department of Conservation, Game Division. 404 p. (Publication 100).

Baumgartner, LL. 1943. Fox squirrels in Ohio. Journal of Wildlife Management 7:193-202.

Brown, LN; McGuire, RJ. 1975. Field Ecology of the exotic Mexican red-bellied squirrel in Florida. American Society of Mammalogists 56(2):405-419.

Cudworth, NL; Koprowski, JL. 2011. Importance of scale in nest-site selection by Arizona gray squirrels. The Journal of Wildlife Management 75(7):1668-1674. 
De Vivo, M; Carmignotto, AP. 2015. Family Sciurudae G. Fischer, 1817. In Patton, JL; Pardiñas, FJ; D'Elía, G (eds.). Mammals of South America, Volume 2: Rodents Chicago and London. The University of Chicago Press, p. $1-48$.

Díaz, C; Tantaleán, M. 2012. Helmintos gastrointestinales en ardillas de nuca blanca (Sciurus stramineus) de vida libre en zoológicos de Lima. The Biologist 10 (Jul-Dic), Suplemento Especial 2: Libro de resúmenes del III Congreso Internacional de Parasitología Neotropical (III COPANEO). "Parasitología Global: Salud, Biodiversidad y Zoonosis".

Edelman, AJ; Koprowski JL. 2005. Selection of drey sites by Abert's squirrels in an introduced population. Journal of Mammalogy 86:12201226.

Edelman, AJ; Koprowski, JL; Bertelsen, SR. 2009. Potential for nest site competition between native and exotic tree squirrels. Journal of Mammalogy 90(1):167-174.

Edwards, JW; Guynn, DC. 1995. Nest characteristics of sympatric populations of fox and gray squirrels. The Journal of Wildlife Management 59(1):103-110.

Emmons, LH; Feer, F. 1997. Neotropical rainforest mammals: a field guide. Chicago, USA. The University of Chicago Press. 396 p.

Eydoux, J.F.T; Souleyet, L.F.A. 1841. Voyage autour du Monde exécuté pendant les anées 1836 et 1837 sur la corvette La Bonite commandée par M. Vaillant. Paris: Arthus Bertrand, 1:xl+132 pp.; Atlas: plates 1-12 (Mamifères).

Farentinos, RC. 1972. Nests of the tassel-eared squirrel. Journal of Mammalogy 53(4):900903.

Geoffroy St.-Hilaire, I1855. Mammifères. In Voyage autour du monde sur La frégate la Vénus, commandee par Abel du Petit-Thouars. Zoologie, mammifères, oiseaus, reptiles et poisons, $1-176+13$ pls. Paris: Gide et J. Baudry, 1-351+i-iii +23 plates.

Gervais, P. 1841. Mammifères. In Eydoux, J.F.T.; L.F.A. Souleyet (eds.) Zoologie., 1-68. In
Voyage autour du Monde execute pendant les annés 1836 et 1837 sur la corvette La Bonite commandée par M. Vaillant. Paris: Arthus Bertrand, 1:xl + 132 pp.; Atlas: plates 1-12 (Mammiferès).

Gurnell, J. 1987. The Natural History of Tree Squirrels. New York, Estados Unidos de América. Facts on File Inc. 224 p.

Halloran, ME; Bekoff, M. 1994. Nesting-behavior of Abert squirrels (Sciurus aberti). Ethology 97(3):236-248.

Jessen, RR. 2013. Behavior and ecology of neotropical tree squirrels in seasonally flooded forests in the Peruvian amazon. Tesis M.Sc., Arizona, Estados Unidos de América. The University of Arizona. 11p.

Jessen, RR; Merrick, MJ; Koprowski, JL; Ramirez, O. 2010. Presence of Guayaquil squirrels on the central coast of Peru: an apparent introduction. Mammalia 74(4):443-444.

Jessen, RR; Palmer, GH; Koprowski, JL. 2013. Maternity nest of an Amazonian red squirrel in a bromeliad. Mastozoología Neotropical 20(1):159-161.

Knick, ST; Rotenberry, JT. 1995. Landscape characteristics of fragmented shrub steppe habitats and breeding passerine birds. Conservation Biology 9:1059-1071.

Korpowski, JL; Nandini, R. 2008. Global hotspots and knowledge gaps for tree and flying squirrels. Current Science 95(7):851-856.

Layne, JN. 1954. The biology of the red squirrel, Tamiasciurus hudsonicus loquax (Bangs) in central New York. Ecological Monograph 24:227-267.

Long, JL. 2003. Introduced Mammals of the World: their history, distribution and influence., Collingwood, Australia. SCIRO Publishing. $612 \mathrm{p}$.

Merrick, MJ; Bertelsen, SR; Koprowski, JL. 2007. Characteristics of Mount Graham red squirrel nest sites in a mixed conifer forest. Journal of Wildlife Management 71(6):19581963. 
Merrick, MJ; Koprowski, JL; Gwinn, RN. 2012. Sciurus stramineus (Rodentia: Sciuridae). Mammalian Species 44(1): 44-50.

Middleton, AD. 1931. The grey squirrel: the introduction and spread of the American grey squirrel in the British Isles, its habits, food and relations with the native fauna of the country. London, Reino Unido. Sidgwick \& Jackson. 107 p.

Montes, D; Rivera, H; Ramírez, M; Ríos, P; Angulo, C; Muñoz, K. 2011. Frecuencia de infección por Leptospira sp. en ardillas nuca blanca (Sciurus stramineus) silvestres en un zoológico de la ciudad de Lima. Revista de Investigaciones Veterinarias del Perú 22(1):66-71.

Pacheco, V; Cadenillas, R; Salas, E; Tello, C; Zeballos, H. 2009. Diversidad y endemismo de los mamíferos del Perú. Revista Peruana de Biología 16:5-32.

Parker, TS; Nilon, CH. 2008. Gray squirrel density, habitat suitability, and behavior in urban parks. Urban Ecosystems 11(3):243-255.

PUCP (Pontificia Universidad Católica del Perú). 2018. Clima de Camabios PUCP: Flora y Fauna PUCP (en línea, sitio web). Consultado 3 set. 2018. Disponible en https://www. pucp.edu.pe/climadecambios/la-pucp-frenteal-cambio-climatico/registro-de-flora-y-fauna-de-la-pucp/.

Ramos-Lara, N; Cervantes, FA. 2007. Nest-site selection by the Mexican red-bellied squirrel in Michoacán, Mexico. Journal of Mammalogy 88(2):495-501.

Raspopov, MP; Isakov, YA. 1980. Biology of the squirrel. New Delhi, India. Amerind Publishing. 56 p. Extracto de Biologiya Zaistev i Belok i ikh Bolezni 1935 (p. 33-77), traducido del ruso y publicado para Forest Service - United States Department of Agriculture and the National Science Foundation.

Rotenberry, JT; Wiens, JA. 1980. Habitat structure, patchiness, and avian communities in North American steppe vegetation: a multivariate analysis. Ecology 61:1228-1250.

Ruiz, AT; Rubín, E; Vásquez, P. 2014. Estrategia de educación ambiental para la mejora de la gestión del zoocriadero de venados en la Pontificia Universidad Católica del Perú. Xilema 27:24-32.

Salsbury, CM. 2008. Distribution patterns of Sciurus niger (eastern fox squirrel) leaf nests within woodlots across a suburban/urban landscape. Northeastern Naturalist 15:485-496.

Sanderson, HR; Healy, WM; Pack, JC; Jill, JD; Thomas, JW. 1976. Gray squirrel habitat and nest tree preference. Proceedings of Annual Conferences of the Southeastern Association Game and Fish Commission 29:609-616.

SERNANP (Servicio Nacional de Áreas Naturales Protegidas, Perú). 2010. Plan de uso turístico del Santuario Histórico Bosques de Pómac. Lima, Perú. ACBT PROFONANPE y ProNaturaleza.

Shorten, M. 1951. Some aspects of the biology of the grey squirrel (Sciurus carolinensis) in Great Britain. Proceeding of the Zoological Society of London 121(2):427-451.

Shorten, S. 1962. Red Squirrels. In Harrison Mathews, L. (ed.) Animals of Britain. The Sunday Times, London. v.6.

Snyder, MA; Linhart, YB. 1994. Nest-site selection by Abert's squirrels: chemical characteristics of nest trees. Journal of Mammalogy 75(1):136-141.

Steele, MA; Koprowski, JL. 2001. North American tree squirrels. Washington. USA. Smithsonian Institution Press. 224 p.

Thorington, RW; Koprowski, JL; Steele, MA; Whatton, JF. 2012. Squirrels of the world. Baltimore, USA. The John Hopkins University Press. 472 p.

Tirira, DG. 2007. Mamíferos del Ecuador: Guía de campo. Quito, Ecuador. Ediciones Murciélago Blanco. 576 p.

Tittensor, A. 1970. Red squirrel dreys. Journal of Zoology 162(4):528-533.

Vahle, JR; Patton, DR. 1983. Red squirrel cover requirements forests in mixed conifer forests. Journal of Forestry 81(1):14-16. 
Williams, E. 2011. A comparison of eastern gray squirrel (Sciurus carolinensis) nesting behavior among habitats differing in anthropogenic disturbance. Tesis M.Sc. Georgia, US. Georgia Southern University. 67 p.

Presentado: 06/10/2018

Aceptado: 18/12/2018

Publicado en línea: 28/12/2018 